\title{
Las sentencias de unificación en materia tributaria. Caso de la tasa de vigilancia del artículo 85 de la Ley 142 de 1994
}

\section{Unification sentences in tax matters Case of the surveillance rate of article 85 of Law 142 of 1994}

\section{Sentença de unificação em matéria tributária Caso da taxa de fiscalização do artigo 85 da Lei 142 de 1994}

Ana María García Galeano*

\footnotetext{
Abogada de la Universidad de Caldas, especialista en Derecho Tributario de la Universidad Externado de Colombia y candidata a la maestría en Derecho del Estado con énfasis en Derecho Público de la misma institución, profesional universitaria de la Sección Cuarta del Consejo de Estado. Correo-e: anamariagarciagaleano@gmail.com. ORCID ID: https://orcid.org/0000-00021651-129X Dor: https://doi.org/10.18601/16926722.n17.07
} 


\section{Resumen}

El presente escrito analiza las debilidades y fortalezas de las sentencias de unificación introducidas por el Código de lo Contencioso Administrativo en el ámbito tributario, en Colombia, emitidas por la Sección Cuarta del Consejo de Estado a partir de un caso tipo. El valor del escrito radica en estudiar la importancia de esta realidad jurídica que sin lugar a duda se relaciona con la seguridad jurídica.

Palabras clave: Sentencias de unificación; Seguridad jurídica; Igualdad.

\section{Abstract}

This document analyzes the weaknesses and strengths of the unification sentences introduced by the Code of Administrative Litigation in the tax field, in Colombia, issued by Section IV of the Council of State from a typical case. The importance of the writing lies in studying the importance of this legal reality that undoubtedly relates to legal certainty.

Keywords: Unification Sentences; Legal Certainty; Equality.

\section{Sumário}

Este documento analisa os pontos fracos e fortes das sentenças de unificação introduzidas pelo Código de Litígios Administrativos no campo tributário, na Colômbia, emitido pela Seção Iv do Conselho de Estado a partir de um caso típico. A importância da escrita reside no estudo da importância dessa realidade jurídica que, sem dúvida, se relaciona com a segurança jurídica.

Palavras-chave: Sentenças de unificação; Segurança jurídica; Igualdade. 


\section{Introducción}

Con miras a abordar la necesidad de sentencias que unifiquen asuntos controvertidos en materia tributaria, se expondrá un caso en el que la jurisprudencia de la Sección Cuarta ha emitido decisiones que, reiterativamente, plantean una misma posición en torno a un problema jurídico específico y, adicionalmente, en el decurso de sus pronunciamientos repetitivos también ha integrado ciertos elementos que estuvieron atados a los asuntos particularmente demandados, pero que, sin lugar a dudas, se proyectaron sobre muchas situaciones jurídicas particulares.

En efecto, el legislador, a través de la Ley 1437 de 2011 (CPACA), previó la sentencia de unificación como un mecanismo judicial y administrativo que, por una parte, propende por integrar en un solo fallo la solución a un problema jurídico por importancia jurídica, trascendencia económica o social o por la necesidad de fijar una posición jurídica vinculante, dado que ese tipo de decisión es emitida por el Consejo de Estado como órgano de cierre de la jurisdicción contencioso-administrativa. Por otra parte, dicha sentencia de unificación irradia a los actos administrativos y al proceso de formación de estos, dado que el artículo 102 del CPACA estatuyó la extensión de los efectos de las sentencias de unificación en casos en los cuales se acrediten los mismos supuestos fácticos y jurídicos sobre los que versó el análisis del fallo que unificó la jurisprudencia. En este último evento, la Administración estará en la obligación de aplicar la sentencia de unificación que no tendrá el mero alcance de un precedente judicial, sino que el elemento integrador y vinculante también lo es para las decisiones administrativas a partir de los criterios fijados por esa jurisprudencia.

Verdaderamente, este mecanismo no solo sirve como decisión que integra la multiplicidad de situaciones fácticas que hayan derivado en varias sentencias, sino que el resultado de su poder vinculatorio tanto a los procesos judiciales como a la propia autoridad, relevará al administrado de tener que demandar los actos administrativos que definan la situación jurídica análoga a la dirimida en el fallo de unificación e, igualmente, el debate se restringirá a la solicitud de la extensión de jurisprudencia y el trámite expedito de la revisión que hará el juez o magistrado respecto del acto que niegue tal extensión. El resultado de esto será que se descongestionarán la administración de justicia en cuanto a procesos ordinarios.

Empero, ese mundo ideal de las sentencias de unificación ha trasegado tímidamente desde su creación con el CPACA -que entró en vigor el 2 de julio de 2012- hasta la fecha. De hecho, la Sección Cuarta del Consejo de Estado no ha emitido aún sentencias de unificación (este escrito fue finalizado el 10 de octubre de 2019), así que esto marca la falta de notoriedad que ha tenido para la administración de justicia ese tipo de fallos, a pesar de su gran utilidad.

Precisamente, uno de los casos que más ha llevado a demandas judiciales trata sobre la tasa de vigilancia del artículo 85 de la LSPD. Dependiendo del sistema de buscador de jurisprudencia de la relatoría del Consejo de Estado, podemos hallar más de 160 sentencias 
relacionadas con la tasa de vigilancia, específicamente, con el problema jurídico asociado a la base gravable de ese tributo.

El lapso de los actos administrativos demandados nos remonta desde la entrada en vigor de la Ley 142 de 1994 y hasta la fecha aún son emitidas decisiones sobre el mismo punto de inconformidad, pues la SuperServicios, que cumple el papel de sujeto activo de la relación jurídico-tributaria, ha insistido en que las decisiones judiciales anteriormente emitidas, desatienden el artículo 338 de la Constitución. Cabe registrar que la Ley 1955 de 2019 modificó el artículo 85 de la LSPD y, con ello, el legislador esquivó la posición jurídica de la Sección Cuarta al mejorar la redacción de la norma que regulaba la base gravable de esa tasa.

Con todo, no se debe perder el interés en el hecho de que la administración de justicia pudo evitar la multiplicidad de juicios ordinarios que derivaron en la réplica de decisiones emitidas con anterioridad. Ese mecanismo hubiese sido la solución no solo a la congestión de los procesos tramitados ante el Consejo de Estado, sino todos aquellos que fueron dirimidos por los jueces y magistrados que hacen parte de la jurisdicción contencioso- administrativa.

\section{Contextualización del debate surgido respecto de la tasa de vigilancia del artículo 85 de la LSPD}

De acuerdo con el artículo 1. o de la Ley 142 de 1994, su ámbito de aplicación es para los servicios públicos de acueducto, alcantarillado, aseo, energía eléctrica, distribución de gas combustible, telefonía fija pública básica conmutada y la telefonía local móvil en el sector rural.

A su turno, los artículos 14 ibídem, 15 y 17, parág. identifican quiénes pueden prestar esos servicios públicos que básicamente podrán conformarse de las siguientes formas: empresas de servicios públicos oficiales, empresa de servicios públicos mixtas, empresas de servicios públicos de naturaleza privada, empresas industriales y comerciales del Estado - tal es el caso de Emcali, EPM y Acueducto de Bogotá-. Según el tipo de forma asociativa que se elija, dicho régimen legal también habilita que la prestación de los servicios públicos domiciliarios sea prestada por: las personas naturales o jurídicas que produzcan asimismo los servicios propios de las empresas de servicios públicos, los municipios que asuman dicha prestación, las organizaciones autorizadas en municipios menores o zonas rurales, las entidades que, de manera temporal, podrán prestar esos servicios y las entidades descentralizadas del orden territorial o nacional que estuvieren prestando servicios públicos domiciliarios a la entrada en vigencia de la LSPD.

De esta forma, las principales entidades sobre las cuales repercuten las regulaciones de la LSPD serán las anteriores personas naturales y jurídicas de Derecho público o privado.

Ahora bien, de conformidad con el artículo 3. ${ }^{\circ}$ de la LSPD, la SuperServicios será la entidad encargada del cumplir el control, inspección y vigilancia, sobre las anteriores entidades que quedarán sujetas a las regulaciones que prevé dicho organismo del orden técnico. 
Y la misma Constitución y ley fijaron los mecanismos de financiación del servicio público de control, inspección y vigilancia que presta dicha superintendencia (artículos 370 de la Carta y 85 de la LSPD). Al efecto:

Artículo 85. Contribuciones especiales. Con el fin de recuperar los costos del servicio de regulación que preste cada comisión, y los de control y vigilancia que preste el Superintendente, las entidades sometidas a su regulación, control y vigilancia, estarán sujetas a dos contribuciones, que se liquidarán y pagarán cada año conforme a las siguientes reglas:

$(\ldots)$

85.2. La superintendencia y las comisiones presupuestarán sus gastos cada año y cobrarán dentro de los límites que enseguida se señalan, solamente la tarifa que arroje el valor necesario para cubrir su presupuesto anual.

La tarifa máxima de cada contribución no podrá ser superior al uno por ciento (1\%) del valor de los gastos de funcionamiento, asociados al servicio sometido a regulación, de la entidad contribuyente en el año anterior a aquel en el que se haga el cobro, de acuerdo con los estados financieros puestos a disposición de la Superintendencia y de las Comisiones, cada una de las cuales e independientemente y con base en su estudio fijarán la tarifa correspondiente.

A partir de la estructuración del tributo como fuente de financiación de la prestación de un servicio por parte de la SuperServicios y, con base en las atribuciones constitucionales y legales, dicho organismo anualmente fija la denominada contribución especial, a cargo de las entidades destinatarias de la LSPD, que también cumplirán el papel de sujeto pasivo de la obligación tributaria.

Si bien la normativa no nos ofrece dificultades interpretativas en torno a los aspectos material, temporal y espacial, no sucede lo mismo respecto de la cuantificación del tributo, pues la norma alude al concepto de "gastos de funcionamiento, asociados al servicio sometido a regulación”. La razón por la que digo que la anterior expresión genera dificultades interpretativas es porque la ley no definió ni acotó el alcance de los llamados gastos de funcionamiento, ello sin perder de vista que las tasas propendan por recuperar los costos en la prestación del respectivo servicio.

Pues bien, la cuantificación del tributo consiste en que, anualmente, la SuperServicios expedirá un acto administrativo general que fijará la tarifa de la tasa, con observancia del límite máximo del $1 \%$ sobre la base gravable, la cual, antes de la modificación del artículo 18 de la Ley 1955 de 2019, consistía en los gastos de funcionamiento del año inmediatamente anterior, según los estados financieros reportados por las empresas prestadoras de servicios públicos domiciliarios al sujeto activo de la relación jurídico-tributaria. Estos gastos de funcionamiento no podrán incluir los gastos operativos. En el caso de las empresas del sector eléctrico no se incluirán las compras de electricidad, compras de combustible y los peajes y, en cuanto a las empresas de otros sectores, no se incluirán 
gastos de naturaleza similar a los anteriores. Sin embargo, en caso de que existan faltantes presupuestarios de la SuperServicios se podrá adicionar la base gravable, en proporción a lo que faltare para cubrir el presupuesto de gastos de funcionamiento de la entidad administrativa, el cual es punto de partida para poder fijar la tarifa aplicable a cada período fiscal (este procedimiento se realiza anualmente).

Para identificar cómo las entidades prestadoras de servicios públicos domiciliarios registrarán en su sistema contable los gastos de funcionamiento, debe precisarse que, según los artículos 79.4 de la LSPD, en concordancia con el 5-13 del Decreto 990 de 2002, la SuperServicios adoptará los sistemas uniformes de información y de contabilidad de las empresas sometidas a su vigilancia y control. Con base en esa normativa, la SuperServicios expidió la Resolución SSPD 1416 de 1997, relativa al Plan de Contabilidad para Entes Prestadores de Servicios Públicos Domiciliarios, norma que fue modificada por las resoluciones SSPD 006572, del 04 de septiembre de 2001 y SSPD 3064, del 01 de marzo de 2002, que, entre otras cuestiones, establecieron que el marco conceptual y el manual de procedimiento del Plan General de Contabilidad Pública -Resolución 400 de 2000 de la Contraloría General de la República- era aplicable a las empresas prestadoras de servicios públicos de naturaleza oficial y mixta.

Según el plan de contabilidad de entes prestadores de servicios públicos, el catálogo de las cuentas en las que se remitían la información estaría agrupado por clase, grupo, cuenta, subcuenta y auxiliar. La clasificación clase obedece a: activos (clase 1), pasivos (clase 2), patrimonio (clase 3), ingresos (clase 4), gastos (clase 5), costos de ventas y de operación (clase 6), costos de producción (clase 7), cuentas de orden deudoras (clase 8) y cuentas de orden acreedoras (clase 9).

En el caso de los rubros pertenecientes a la clase 5 (gastos), la normativa que inicialmente reguló el plan de contabilidad para los entes prestadores de servicios públicos definió los gastos conforme a lo previsto en la Resolución 400 de 2000, que, como se dijo, corresponde al Plan de Contabilidad Pública expedido por la CGR. Tal acepción prescribía que los gastos eran "flujos de salida de recursos de la entidad contable pública, susceptibles de reducir el patrimonio público durante el período contable, bien sea por disminución de activos o por aumento de pasivos, expresados en forma cuantitativa". Al mismo tiempo, la definición precisó que también son gastos aquellos requeridos para el desarrollo de la actividad ordinaria, incluidos los originados por situaciones de carácter extraordinario.

Ahora bien, como quiera que el artículo 85 de la LSPD establecía que la base gravable de la tasa de vigilancia estaría conformada por los "gastos de funcionamiento asociados a la prestación del servicio público" y, dado que la tasa propendía por financiar la actividad administrativa de inspección, vigilancia y control de la Superintendencia, tal entidad asumió que los gastos de funcionamiento no solamente hacían alusión a la noción técnica contable de gastos, sino a todos aquellos rubros empleados por la entidad prestadora del servicio público domiciliario que servía para desarrollar tal actividad económica. Por ello, la SuperServicios, desde la expedición del Plan de Contabilidad para Entes Prestadores 
de Servicios Públicos Domiciliarios -Resolución 1416 de 1997-, asumió que la noción de gastos de funcionamiento no solamente incluía a los conceptos registrados en la clase 5 (gastos), sino también algunos de la clase 7 (costos de producción), dado que ambos rubros constituían egresos que le permitían a la respectiva empresa la prestación del servicio público domiciliario.

Al efecto, dentro de la clase 7 de costos de producción, la SuperServicios los clasificó en los siguientes grupos:

- 71 Producción de bienes.

- 72 Servicios educativos.

- 73 Servicios de salud.

- 74 Servicios de transporte.

- 75 Servicios públicos.

- 76 Servicios hoteleros y de promoción turística.

- 79 Otros servicios.

Además, definió dichos costos, así:

En esta denominación se incluyen los grupos que representan las erogaciones y cargos directamente relacionados con la producción de bienes y la prestación de los servicios individualizables que surgen del desarrollo de funciones de cometido estatal de la entidad contable pública, con independencia de su suministro gratuito o su venta a precios económicamente no significativos o a precios de mercado. También incluye el costo de los bienes producidos para el uso o consumo interno. Los costos de producción están asociados principalmente con la obtención de los ingresos o parte de ellos, por la venta de bienes o la prestación de servicios individualizables. Las cuentas que integran esta clase son de naturaleza débito. Cuando se trate de la producción de bienes que serán consumidos o utilizados directamente por la entidad contable pública, una vez concluido el proceso productivo, deben reclasificarse a las cuentas de gastos, cargos diferidos, propiedades, planta y equipo o al activo correspondiente. (...)

Siendo que la definición de costos de producción sí se podía asociar directamente a la prestación del respectivo servicio público, el sujeto activo de la relación jurídico-tributaria determinó que hacía parte de la base gravable de la tasa de vigilancia. Tal consideración fue también incluida en la Resolución 25 de 1998, que expidió el formulario de autoliquidación de la tasa de vigilancia. Asimismo, en los sucesivos actos administrativos que fijaron anualmente la tasa de vigilancia fueron incluidas algunas de las cuentas de la clase 7 (costos de producción), específicamente del grupo 75 (servicios públicos).

Adicionalmente, también la SuperServicios incluyó, en algunos casos, gastos del grupo 53 (provisiones, agotamiento, depreciaciones, y amortizaciones), particularmente, las siguientes cuentas: 5304 (provisión para deudores), 5313 (provisión para obligaciones 
fiscales), 5330 (depreciación de propiedades, planta y equipo), 5331 (depreciación de bienes adquiridos en leasing financiero) y 5345 (amortización de intengibles). Asimismo, adicionó el grupo 51 (gastos por pensiones), la cuenta 5120 (impuestos, tasas y contribuciones) y la cuenta 5810 (otros gastos extraordinarios).

\section{A. La definición jurisprudencial de los gastos de funcionamiento y la oposición de la Administración}

Como se explicó en la contextualización del problema jurídico resuelto en las múltiples decisiones judiciales, la SuperServicios decidió incluir en la noción de gastos de funcionamiento aquellos costos de producción que se relacionaban directamente con la prestación del servicio público respectivo (grupo 75). De igual forma, incluyó algunas cuentas pertenecientes a la clase 5 (gastos). Sin embargo, muchos de los actos administrativos que decidieron incluir estos rubros fueron objeto de demandas que determinaron la nulidad parcial de las disposiciones que incluyeron ciertas cuentas que, según la jurisprudencia, no podían asociarse a la prestación del servicio sometido a vigilancia o no concatenaban con la definición contable de gastos.

En el proceso 11790 se demandó la nulidad del artículo 5. ${ }^{\circ}$ de la Resolución 25 de 1998, acto que expidió el formulario de autoliquidación de la contribución especial -tasa de vigilancia-. El 09 de noviembre de 2001 fue expedida la sentencia que anuló dicha disposición y que contribuyó en la delimitación de la noción de gastos de funcionamiento, prevista en el artículo 85 de la LSPD (sentencia del 09 de noviembre de 2001, exp. 11790, C.P.: María Inés Ortiz Barbosa).

En esa oportunidad, dicha corporación definió los gastos de funcionamiento, así:

La expresión "funcionamiento" significa: Acción y efecto de funcionar. Funcionar. Ejecutar una persona, máquina, etc. Las funciones que le son propias. De donde se deduce que los Gastos de Funcionamiento son aquellos flujos de salida de recursos que generan disminuciones del patrimonio realizados para ejecutar o cumplir las funciones propias de su actividad, lo que en términos técnico contables simbolizan los Gastos Operacionales u Ordinarios, es decir los normalmente ejecutados dentro del objeto social principal del ente económico.

Particularmente, la decisión recayó sobre los gastos por pérdida en venta o baja de activos fijos que incluyó el formulario de autoliquidación de la tasa. De acuerdo con la definición que estableció dicha corporación, se concluyó que la pérdida en venta o baja de activos no es un gasto "normal ni ordinario" efectuado por una empresa prestadora de servicios públicos y, por ello, no podía hacer parte de la base gravable del tributo.

Esta definición fue reiterada por múltiples providencias, tales como las proferidas en expedientes identificados con números: 16650 (auto del 24 de noviembre de 2007, C.P.: Juan Ángel Palacio Hincapié), 16841 (auto del 24 de noviembre de 2007, C.P.: María Inés 
Ortiz Barbosa), 15771 (sentencia del 17 de abril de 2008, c.P.: María Inés Ortiz Barbosa), 17206 y 17159 (sentencias del 04 de febrero de 2010, c.P.: William Giraldo Giraldo), 16874 (sentencia del 23 de septiembre de 2010, c.P.: Martha Teresa Briceño de Valencia). A propósito de estas decisiones, vale la pena mencionar que las sentencias del 17 de abril de 2008 (exp. 15771) y del 23 de septiembre de 2010 (exp. 16874) contribuyeron a afianzar la definición de gastos de funcionamiento. De hecho, la decisión emitida en el expediente 16874 ha sido invocada en los fallos que todavía se emiten, a pesar de que la sentencia fundadora de la definición de gastos de funcionamiento correspondió a la del 09 de noviembre de 2001 (exp. 11790, c.P.: María Inés Ortiz Barbosa).

En relación con la sentencia del 17 de abril de 2008 (exp. 15771, C.P.: María Inés Ortiz Barbosa), el Consejo de Estado nuevamente reiteró la acepción de gastos de funcionamiento ya indicada desde el fallo del año 2001; pero, adicionalmente, precisó que dichos gastos no se restringían a los directamente relacionados con la prestación del servicio sometido a vigilancia, sino también a aquellos que atendían a las necesidades de esas empresas. Al respecto:

Cuando el artículo 85.2 de la Ley 142 de 1994 señala que la base para liquidar la contribución es el valor de los gastos de funcionamiento asociados al servicio sometido a regulación, no se refiere solamente a aquellos gastos que están directamente dirigidos al desarrollo de la operación básica o principal de la entidad, pues estos son los gastos de operación definidos así, por el Sistema Nacional de Contabilidad Pública, sino a los gastos que aunque no estén directamente relacionados con la prestación del servicio u operación básica, sí le son concernientes. Los gastos de funcionamiento son aquellas erogaciones que tienen por objeto atender las necesidades de las entidades para cumplir a cabalidad con las funciones asignadas en la Constitución Política y en la Ley; y una de esas necesidades es cumplir con la carga pensional de sus trabajadores, pues los gastos de personal son las erogaciones que debe hacer el Estado como contraprestación de los servicios que recibe, ya sea por una relación laboral o a través de contrato, lo cual incluye, tal como reconoce el actor, garantizar el amparo contra las contingencias derivadas de la vejez, la invalidez y la muerte, mediante el reconocimiento de las pensiones y prestaciones que determina la ley 100 de 1993.

Por su parte, el fallo del 23 de septiembre de 2010 (exp. 16874, C.P.: Martha Teresa Briceño de Valencia) acudió a la definición de gastos de funcionamiento que ya había fijado la decisión del 09 de noviembre de 2001:

Aunque el Diccionario de Términos Contables contenido en el Plan de Contabilidad para Entes Prestadores de Servicios Públicos Domiciliarios define los "gastos de funcionamiento" como "Erogaciones que tienen por objeto atender las necesidades de los órganos para cumplir a cabalidad con las funciones asignadas en la Constitución Política y la ley", este concepto resulta amplio e impreciso para efectos de determinar la 
base gravable de la contribución especial. Por lo tanto, será necesario acudir a otros elementos conceptuales para encontrar un criterio auxiliar que ayude en la interpretación del término "gastos de funcionamiento". Esta Corporación ha tenido la oportunidad de pronunciarse sobre el alcance del término "gastos de funcionamiento" en la sentencia del 9 de noviembre de 2001, Expediente 11790, m.P.: Dra. María Inés Ortiz Barbosa. Por tanto, ante la ausencia de normativa contable que defina con claridad lo que debe entenderse por el término "gastos de funcionamiento" y los conceptos que involucra, la Sala acogerá la noción que ha desarrollado la jurisprudencia y, en consecuencia, el presente proceso se decidirá bajo tales parámetros, es decir, que los gastos de funcionamiento tienen que ver con la salida de recursos que de manera directa o indirecta se utilizan para ejecutar o cumplir las funciones propias de la actividad, que son equivalentes a los gastos operacionales u ordinarios, es decir los normalmente ejecutados dentro del objeto social principal del ente económico o, lo que es lo mismo, los gastos asociados al servicio sometido a regulación, de manera que deberán excluirse aquellas erogaciones que no estén relacionadas con la prestación del servicio público domiciliario.

Con base en esos criterios fijados, la jurisprudencia continuó reiterando dicha posición, pues, a pesar de que la Sección Cuarta ha mantenido tal definición, por su parte, la SuperServicios ha insistido en que la tasa de vigilancia debe propender por recuperar todos los costos en que incurre el sujeto activo de la relación jurídico-tributaria, conforme lo establece el artículo 338 constitucional, de modo que el artículo 85 de la LSPD no podía contravenir la Carta, so pena de quebrantar el artículo 4 de la misma. Con base en esa posición la SuperServicios ha solicitado que oficiosamente se inapliquen las disposiciones legales a fin de que no se restrinja la base gravable de la tasa.

Empero, el razonamiento de la Administración no ha persuadido a la jurisdicción contencioso-administrativa y, en cambio, se ha replicado la jurisprudencia en muchísimas decisiones. Baste con mencionarse las siguientes: sentencias del 14 de octubre de 2010 (exp. 16650, C.P.: Hugo Fernando Bastidas Bárcenas), 26 de enero de 2012 (exp. 16841, C.P.: Martha Teresa Briceño de Valencia), 01 de noviembre de 2012 (exps. 17441 y 17515, C.P.: Hugo Fernando Bastidas Bárcenas), 29 de noviembre de 2012 (exp. 18862, C.P.: Martha Teresa Briceño de Valencia), 21 de mayo de 2014 (exp. 18746, C.P.: Hugo Fernando Bastidas Bárcenas), del 28 de agosto de 2014 (exp. 20002, c.P.: Jorge Octavio Ramírez Ramírez), 05 de febrero de 2015 (exp. 18067, c.P.: Carmen Teresa Ortiz de Rodríguez), del 05 de marzo de 2015 (exp. 20485, c.P.: Martha Teresa Briceño de Valencia), 02 de julio de 2015 (exp. 21032, c.P.: Martha Teresa Briceño de Valencia), 10 de septiembre de 2015 (exp. 21254, c.P.: Jorge Octavio Ramírez Ramírez), 24 de septiembre de 2015 (exp. 19146, C.P.: Carmen Teresa Ortiz de Rodríguez), auto del 10 de febrero de 2016 (exp. 21605, C.P.: Jorge Octavio Ramírez Ramírez), 25 de abril de 2013 (exp. 18931, C.P.: Carmen Teresa Ortiz de Rodríguez), 30 de agosto de 2016 (exp. 21442, C.P.: Martha Teresa Briceño de Valencia), auto del 07 de octubre de 2016 (exp. 19762, C.P.: Martha Teresa Briceño de Valencia), 08 de noviembre de 2017 (exp. 22820, C.P.: Milton Chaves García), 
13 de diciembre de 2017 (exps. 21855 y 22949, c.P.: Julio Roberto Piza Rodríguez), 12 de diciembre de 2018 (exps. 22381 y 23012, C.P.: Julio Roberto Piza Rodríguez), auto del 24 de enero de 2019 (exp. 22394, c.P.: Jorge Octavio Ramírez Ramírez), 12 de febrero de 2019 (exp. 23462, C.P.: Stella Jeannette Carvajal Basto), del 14 de marzo de 2019 (exp. 23125, C.P.: Julio Roberto Piza Rodríguez), del 14 de marzo de 2019 (exp. 24086, C.P.: Julio Roberto Piza Rodríguez) y, 02 de mayo de 2019 (exp. 22161, C.P.: Stella Jeannette Carvajal Basto $)^{1}$.

Las anteriores son solo algunas de las decisiones que se han proferido en torno al mismo problema jurídico relacionado con si las cuentas de gastos, previstas en el acto administrativo anual que liquida la tasa de vigilancia, concatenan con la definición de gastos de funcionamiento que brindó la Sección Cuarta desde el fallo del 09 de noviembre de 2001.

Más allá de si la definición se corresponde con el espíritu del legislador a la hora de redactar el artículo 85.2 de la LSPD, lo cierto es que ha sido un desgaste administrativo y judicial frente a una misma situación jurídica que se pudo finiquitar con una sentencia de unificación, por lo menos frente a procesos ordinarios judiciales, pues este era uno de los precisos casos que limitaría la reproducción del acto anulado como fue lo que sucedió durante más de una década en este tema específico.

Si bien la definición de gastos de funcionamiento que dio la jurisprudencia no resolvió, plenamente, las discrepancias entre la posición de los contribuyentes y la de la Administración, en tanto que esta última tenía la necesidad de recuperar los costos del servicio de inspección, control y vigilancia. Además, parte de la terquedad de la entidad demandada devino en que la definición jurisprudencial dejó por fuera a los costos de producción del grupo 75 del PUC para empresas prestadoras de servicios públicos, la razón se afincó en que, a pesar de que esos costos estaban relacionados con la actividad sometida a inspección y vigilancia, lo cierto era que el legislador limitó los recursos que harían parte de la base gravable de la respectiva tasa al señalar en el artículo 85.2 de la mencionada ley que hacían parte los gastos, así que, al no hacer referencia a costos estos no se podían incluir para la determinación de la cuota tributaria.

No obstante, una sentencia de unificación que revisara pormenorizadamente la definición dada por la jurisprudencia quizás hubiera evitado la dilación de un problema jurídico a tal punto que fue el propio legislador en la Ley 1955 de 2019 el que eliminó la expresión de gastos de funcionamiento, en aras de que se pudieran incluir los costos asociados a la prestación del servicio público correspondiente.

1 Estas son solo apenas unas de las muchas que se han emitido por parte de la Sección Cuarta del Consejo de Estado. 


\section{Procedencia de la sentencia de unificación ante demandas recurrentes en la jurisprudencia contencioso-administrativa}

Como trató de ponerse de presente, han sido varias las decisiones que repetidamente han anulado los actos administrativos que fijan la tasa de vigilancia o liquidan el tributo discutido, sin que se hubiere finiquitado la multiplicidad de demandas que recaigan en el mismo punto de inconformidad con el ordenamiento jurídico.

Si bien muchas de las decisiones corresponden a los años en que estuvo vigente el Decreto 01 de 1984 (años 2001 y hasta el 02 de julio de 2012), a partir de la entrada en vigencia de la Ley 1437 de 2011 -CPACA- el legislador introdujo la figura de la sentencia de unificación y de la extensión de la jurisprudencia que tiene un impacto en la concreción del principio de seguridad jurídica -necesario en una jurisprudencia que en varias ocasiones es divergente y confunde la actuación de la Administración y los administrados- y, además, servirá de gran manera a evitar la multiplicidad de procesos que recaen sobre un mismo problema jurídico, ya que la idea de la extensión de la jurisprudencia es evitar un proceso judicial y que sea la propia Administración la que defina una situación jurídica concreta en atención a lo definido en una sentencia de unificación (art. 102 del CPACA). Esto, sin desconocer que la unificación de la jurisprudencia es una función constitucional.

De acuerdo con el artículo 270 ibídem, la sentencia de unificación es emitida por el Consejo de Estado en atención a las siguientes causales: (i) por importancia jurídica; (ii) por trascendencia económica o social, o (iii) por necesidad de unificar o sentar jurisprudencia. Asimismo, califican como sentencias de unificación las que deciden recursos extraordinarios de revisión y revisión eventual de las providencias sobre acciones populares o de grupo.

Los procesos, tramitados en única o segunda instancia ante los tribunales administrativos o, en las mismas instancias, asignados a las secciones o subsecciones del Consejo de Estado y que estén pendientes de fallo, podrán ser decididos mediante sentencia de unificación a petición de parte, por solicitud del Ministerio Público o de oficio (art. 271 ídem).

La función constitucional de la unificación de jurisprudencia propende por afianzar el derecho de la igualdad de quienes acceden a la administración de justicia. En la jurisdicción contencioso-administrativo uno de los primeros antecedentes de la unificación surgió con el recurso extraordinario de súplica, que propendía porque las decisiones de las salas del Consejo de Estado se acompasaran con los pronunciamientos de la Sala Plena de esa corporación (art. 130 antes de la subrogación de la Ley 446 de 1998 y de la derogación de la Ley 954 de 2005). Dicho mecanismo no pretendía desconocer la autonomía e independencia de los jueces, a la luz del artículo 228 constitucional, como tampoco es un objetivo de la actual regulación de la sentencia de unificación y de la extensión de la jurisprudencia.

Por su parte, la Corte Constitucional ha afincado la preponderancia de la unificación en la interpretación de la ley y la aplicación del Derecho (sentencias C-104 de 1993, C-836 de 2001, C-335 de 2008, entre otras). 
La Comisión de Reforma a la Jurisdicción Contenciosa Administrativa, creada mediante el Decreto 4820 de 2007, estableció como propósito que las sentencias de unificación fueran obligatorias para la Administración, de tal forma que la aplicación del Derecho fuera uniforme en cuanto a situaciones fácticas y jurídicas análogas a las dirimidas en providencias de unificación. Al respecto, la exposición de motivos del proyecto de ley 198 de 2009 adujo lo siguiente:

En relación con la garantía del respeto a las decisiones judiciales

Por último, cabe destacar la intención del proyecto en cuanto al acatamiento de las decisiones judiciales, como una manifestación del Estado de Derecho. Por ello, la preocupación de la Comisión se centró en dos aspectos, a saber: uno, el respeto a las decisiones judiciales frente a casos similares y dos, el cumplimiento de las decisiones judiciales.

Para garantizar el respeto a las decisiones judiciales que constituyen jurisprudencia reiterada o de unificación, se propone como mecanismo el derecho a solicitar la extensión y adaptación de la jurisprudencia del Consejo de Estado, contenida en un fallo de unificación jurisprudencial en el que se haya reconocido una situación jurídica, siempre que, en lo pretendido exista similitud de objeto y causa con lo ya fallado ${ }^{2}$.

Está proscrito el trato desigual injustificado a tal punto que la Administración y los jueces deben dar el mismo tratamiento a quienes invoquen precedentes vinculantes, como es el caso de las sentencias de unificación.

Sin perjuicio de lo indicado, en el caso de la tasa de vigilancia de la SuperServicios lo relevante consiste en que la sentencia de unificación hubiese servido como mecanismo que evitara la multiplicidad de decisiones que reiteraron el mismo planteamiento jurídico, en torno a la base gravable del tributo. En esta ocasión, fuerza concluir que desde la entrada en vigor de la Ley 1437 de 2011, la Sección Cuarta del Consejo de Estado ha evitado emitir este tipo de providencias $y$, en cambio, ha preferido continuar emitiendo decisiones que recaban sobre el mismo problema jurídico. Insisto en que la definición de gastos de funcionamiento no necesariamente debe restringirse a la plasmada desde la sentencia del 09 de noviembre de 2001; sin embargo, en la medida en que ha sido un criterio uniforme, era necesario unificar la posición de la Sala, a fin de que la Administración, anualmente, siguiera contrariando las sentencias de anulación.

\section{Conclusiones}

En primer lugar, las decisiones en torno a la denominada contribución especial del artículo 85 de la Ley 142 de 1994 no se concentraron en un fallo unificador que, preferiblemente, 
profundizara acerca de la tipología del tributo e interpretara la norma sobre la cuantificación del tributo en armonía con el regulado.

La solución que estableció la Sección Cuarta frente a la falta de concreción del concepto gastos de funcionamiento fue interpretarlo, dejando por fuera a los costos, en tanto que dentro de la técnica contable no se pueden aparejar a los gastos. Más allá de que esta haya sido la solución correcta a los casos, lo cierto es que ha sido la posición uniforme y, por ello, bien hubiera valido la pena que dicha corporación unificara mediante sentencia, para evitar que la Administración insistiera en contradecir lo ya fijado jurisprudencialmente.

\section{Referencias}

\section{Libros}

Montaña Plata, Alberto (2010). Régimen de los Servicios Públicos Domiciliarios. Bogotá: Universidad Externado de Colombia.

Macho, Luis Míguez (1999). Los Servicios Públicos y el Régimen Jurídico de los Usuarios. Barcelona: CEDECS SL.

Calderón Rivera, Camilo (1998). Planeación Estatal y Presupuesto Público. Bogotá: LEGIS.

Restrepo, Juan Camilo (2014). Derecho Presupuestal Colombiano. Bogotá: LEGIS.

Plazas Vega, Mauricio A. (2000). Derecho de la Hacienda Pública y Derecho Tributario. Bogotá: TEMIS.

ICDT (1999). Derecho Tributario, II edición. Bogotá: ICDT Artículos.

Molina Torres, José Antonio (2003). El reto de construir una definición más comprensiva de la dinámica del servicio público. En: Revista n ${ }^{\circ}$. 1, de Derecho Administrativo de la Asociación Colombiana de Especialistas en Derecho Administrativo (ACESDAT).

Sala de Consulta y Servicio Civil del Consejo de Estado, Namén Vargas, Álvaro. (2014). El mecanismo de extensión de la Jurisprudencia a partir de la entrada en vigencia de la Ley 1437 de 2011. En: Las sentencias de unificación jurisprudencial y el mecanismo de extensión de la jurisprudencia. Bogotá: Ministerio de Justicia y del Derecho.

Atienza, Manuel (1995). Tras la Justicia. Una introducción al Derecho y al Razonamiento Jurídico. Barcelona: Editorial Ariel S. A. 
Bernal Pulido, Carlos (2009). El Neoconstitucionalismo y la Normatividad del Derecho. Bogotá: Universidad Externado, ISBN 978-958-710-429-5.

Laurenz, Karl (2001). Metodología de la Ciencia del Derecho. Barcelona: Edit. Ariel S. A.Ñ, ISBN: 9788434432185.

López Medina, Diego Eduardo (2006). El Derecho de los Jueces obligatoriedad del precedente constitucional, análisis de sentencias y líneas jurisprudenciales y teoría del derecho judicial. Bogotá: Universidad de los Andes, Edit. LEGIS, ISBN 9586534928, 9789586534925 .

\section{Jurisprudencia}

\section{Corte Constitucional}

C-104 de 1993.

C-253 de 1996.

C-483 de 1996.

C-066 de 1997.

C-558 de 2001 .

C-836 de 2001.

C-335 de 2008 .

\section{Consejo de Estado (Sección Cuarta)}

Sentencia del 9 de noviembre de 2001, c.P.: María Inés Ortiz Barbosa, exp. 11790. Definió el concepto de gastos de funcionamiento.

Sentencia del 17 de abril de 2008, c.P.: María Inés Ortiz Barbosa, exp. 15771. Reiteró la definición de gastos de funcionamiento.

Sentencia del 21 de noviembre de 2007, c.P.: Ligia López Díaz, exp. 15851. Definió la tasa de vigilancia de la Superintendencia de Puertos y Transporte como una contribución especial. 
Sentencia del 26 de enero de 2012. C.P.: Martha Teresa Briceño de Valencia, exp. 16841.

Sentencia del 13 de junio de 2013, C.P.: Carmen Teresa Ortiz de Rodríguez (E), expediente 18828.

Sentencia del 20 de junio de 2013, C.P.: Carmen Teresa Ortiz de Rodríguez (E), expediente 18930.

Sentencia del 3 de julio de 2013, C.P.: Carmen Teresa Ortiz de Rodríguez (E) expediente 19017.

Sentencia del 26 de febrero de 2014, C.P.: Jorge Octavio Ramírez Ramírez, (exp. 19155).

Sentencia del 10 de abril de 2014, C.P.: Jorge Octavio Ramírez Ramírez, (exp. (19054).

Auto del 28 de julio de 2017. c.P.: Milton Chaves García, (exp. 21286), providencia que decretó la suspensión provisional de los efectos jurídicos de la Resolución nro. ssPD- 20131300029415 de $1 .^{\circ}$ de agosto de 2013 (la cual fijó la base y la tarifa de la contribución especial para el año 2013). 